\title{
How periodontal disease may contribute to cardiovascular disease
}

\author{
Denis F. Kinane \& Gordon D. O. Lowe
}

Coronary artery disease is the major cause of premature death among men in industrialized countries, and its pathological basis is atherosclerosis. Hemostatic and rheological variables are associated with both prevalent and incident cardiovascular disease and may be mechanisms through which risk factors such as smoking, hyperlipidemia and infections (including periodontal disease) may promote vascular events. Rheological variables (which influence blood flow) are consistently associated with both prevalent and incident cardiovascular disease, possibly because they play a role in localizing atherosclerosis and thrombosis, as well as promoting ischemia. The associations of whole-blood viscosity with cardiovascular disease are partly explained by plasma viscosity and partly by hematocrit. White cell count, but not platelet count, predicts ischemic heart disease events. Cigarette smokers have higher levels of all rheological variables than nonsmokers; these increases are partly or wholly reversible in ex-smokers. Other risk factors such as blood lipids and blood pressure also influence rheological variables. Among hemostatic variables, fibrinogen, factor VIII, von Willebrand's factor complex, tissue plasminogen activator antigen and fibrin $\mathrm{D}$-dimer are associated with both prevalent and incident cardiovascular disease. These findings suggest that endothelial disturbance and increased fibrin turnover may play roles in cardiovascular disease.

The association between dental infections and cardiovascular disease has been reviewed recently and will not be covered here (2). The multifactorial causation of cardiovascular disease and the many risk factors and associations attributed to this condition are shown in Fig. 1 (27). Periodontitis and atherosclerosis have many potential pathogenic mechanisms in common. Both diseases have complex causation, genetic and gender predispositions, and potentially share many risk factors, the most significant of which may be smoking status. The objective of this chapter is to consider the mechanisms whereby a disease such as periodontitis, which is a chronic inflammation initiated by microbial plaque, can predispose to atherosclerosis. Independent risk factors for atherosclerosis and its consequences include age, male gender, smoking, hypercholesterolemia, systemic hypertension, plasma fibrinogen, white cell count, hematocrit and diabetes mellitus $(24,37)$ all of which are also associated with periodontal disease, with the exception of hypercholesterolemia and systemic hypertension $(2,24)$.

As with chronic infections elsewhere in the human body, chronic periodontal infections are associated with systemic changes to the blood and bloodforming organs. For example, a case-control study in Glasgow (23) observed significant increases in plasma fibrinogen concentration and in white blood cell count in patients with chronic gingivitis and periodontitis. These increases correlated with stan-

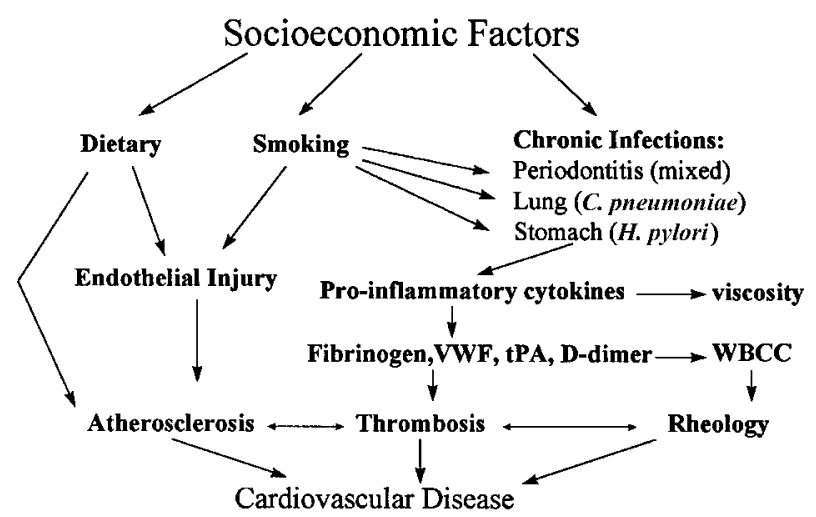

Fig. 1. Risk factors involved in cardiovascular disease. VWF: von Willebrand factor. tPA: tissue plasminogen activator. WBCC: white blood cell count. 
dard indices of infection severity, and were not attributable to group differences in confounding variables such as smoking habit or social class (23). Other common chronic infections, such as Helicobacter pylori gastritis and Chlamydia pneumoniae pneumonitis, have also been associated with increased risk of ischemic heart disease and with increased plasma levels of fibrinogen and of other acute-phase reactant proteins such as C-reactive protein $(33,41)$. Hence, it has been postulated that changes to the blood and blood-forming organs such as hyperfibrinogenemia may be common mechanisms through which infections may promote increased risk of ischemic diseases $(23,33,42)$.

\section{Pathological processes underlying ischemic events}

Four processes may be relevant when considering links between variation in the blood and bloodforming organs and ischemic events: atherosclerosis; arterial thrombosis at the site of a ruptured atherosclerotic plaque; thromboembolism; and the effect of blood viscosity (the intrinsic flow resistance of blood) distal to an atherothrombotic stenosis/occlusion or to an embolic occlusion (Fig. 1).

Atherosclerosis is the focal thickening of arterial intima and media, which tends to occur at bifurcations and bends; that is, sites of flow disturbance where non-laminar or turbulent flow conditions are encountered. These cellular reactions may be a response to endothelial injury, mediated by cytokines and growth factors (40). Infections (including periodontal, H. pylori and C. pneumoniae infections) may be one cause of such injury; as may smoking hypertension, hyperlipidemia, homocysteinemia and oxidant stress.

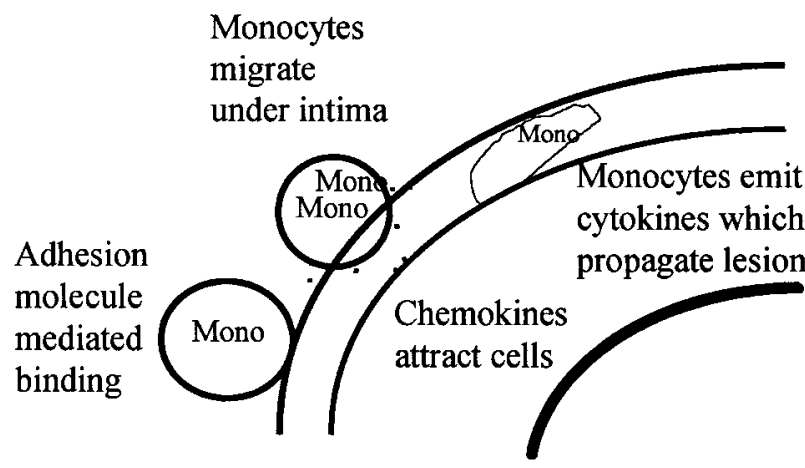

Fig. 2. Monocyte adherence to endothelium, followed by migration between endothelia attracted by chemokines and then deposition of monocyte in subintimal region
Arterial thrombosis often occurs after rupture of an atheromatous plaque, which exposes flowing blood to subendothelial collagen and other fibers (which activate platelets) and to subendothelial tissue factor. The importance of thrombosis in precipitating these ischemic events has been shown by the beneficial effects of antiplatelet drugs (such as aspirin), anticoagulant drugs (such as heparin) or thrombolytic drugs (such as streptokinase or tissue plasminogen activator) in large randomized controlled trials $(8,18)$.

Thromboembolism resulting in systemic arterial occlusion is also a common cause of stroke or critical limb ischemia.

Increased blood viscosity is determined by the composition of the blood: hematocrit; plasma protein pattern; and red cell deformability by shear forces.

The importance of increased blood viscosity in the promotion of ischemia has been demonstrated by the increased risk of ischemic and thrombotic events in the uncommon overt hyperviscosity states such as polycythemia (increased hematocrit due to excess red blood cell production) and the fact that rapid reduction in viscosity (such as by apheresis procedures) may cause rapid symptomatic improvement (26).

\section{Initiation of atherosclerosis}

The initiation of atherosclerotic plaques is thought to be the focal accumulation of lipids (cholesterol esters derived from low-density lipoproteins) and other plasma proteins in the arterial intima (49). The role of monocyte-derived macrophages in this development has become increasingly clear (32). Several studies indicate that monocytes adhere to and penetrate the endothelium during the early stages of atherosclerotic plaque formation (39), aided by adhesion molecules, chemotactic cytokines (chemokines) and proinflammatory cytokines (Fig. 2). These cells become engorged with oxidized low-density lipoproteins and so produce the characteristic foam cells (21). Monocytes transform to macrophages within the lesions, which may cause further destruction by releasing enzymes (11) leading to intramural thrombosis and/or they may enlarge the plaque by releasing mitogenic factors such as platelet-derived growth factor. Fibrin degradation products appear to be involved in both the intramural thrombotic events and have chemotactic and mitogenic properties $(44,46)$ that will further propagate the lesion and lead to smooth muscle cell proliferation, which produces a fibro- 
muscular cap beneath the endothelium and subsequently ischemia $(4,6)$. Adherence of blood monocytes to the vascular endothelium, followed by their migration beneath the intimal endothelium, are considered initiating events in the formation of the characteristic foam cells of atheromas (Fig. 2).

\section{Smoking}

Hemostatic and rheological variables are risk factors for cardiovascular disease and these variables correlate with smoking (27) (Fig. 1). Furthermore, smoking is increasingly accepted as a risk factor for periodontitis and has been shown to affect various aspects of the host immune response (1).

From a biological viewpoint, smoking may have an adverse effect on fibroblast function (38), chemotaxis and phagocytosis by neutrophils $(20,22)$, immunoglobulin production $(17,19)$ and induction of peripheral vasoconstriction (7). Healing responses are impaired in smokers, and this phenomenon might be related to the reportedly impaired function of fibroblasts in smokers (38). Thus smoking, as well as systemic and peripheral infections (which will be discussed subsequently), may produce endothelial irritation through noxious agents, antigenic effects and/or stimulation of proinflammatory cytokines, and these may affect the host inflammatory and immune systems adversely, causing disease. Smoking therefore has potential pathogenic properties that may result in both atherosclerosis and periodontal disease, and smoking is recognized as a shared risk factor or confounding variable given the links between smoking and indices of socioeconomic deprivation. One point worth emphasizing is that the lifestyles of smokers may be different, and their ability to ignore public health messages (as well as the biological effects of smoking) may predispose them to cardiovascular disease through poor diet, lack of exercise and other unhealthy lifestyle practices. The same individuals may also be prone to ignoring dental health advice, which may result in poor oral hygiene practices and thus an exaggerated periodontal disease process.

\section{Infectious diseases}

There is now extensive literature suggesting that cardiovascular disease may be related to infectious diseases (10). The pathological changes noted in ischemic vascular disease have considerable overlap with those caused by a range of infections, including periodontal disease. The question of whether infection can predispose to atherosclerosis is still unanswered, but certainly viral and bacterial pathogens have been considered to play either a direct or indirect role in the initiation of atherosclerosis. With respect to viruses, the link with cytomegalovirus appears to be worthy of discussion (29). Cytomegalovirus has been noted to have an affinity for vascular endothelium (30) and in particular atheromatous plaques (45) where it can be detected by messenger RNA (mRNA) in situ hybridization techniques. Similarly herpes virus inclusions and herpes mRNA have been detected in the intima cells and atheromatous plaques $(3,15)$, where they may cause accumulation of low-density lipoproteins (31).

C. pneumoniae is an intracellular gram-negative bacterium which is known to survive within macrophages for years (9). Saikku et al. (41) found that high antibody titers against $C$. pneumoniae were associated with chronic coronary artery disease and acute myocardial infarction. In addition, Candida albicans has recently been demonstrated in vitro to induce proinflammatory cytokines and adhesion molecule upregulation on endothelial cells (12). These pathogenic features may enhance atheromatous plaque formation in candidal bacteremia, as they would contribute to leukocyte recruitment under the intima.

Thrombogenesis is intimately related to atherogenesis, and hypercoagulable or pro-thrombotic states are considered to predispose to ischemic disease. Fibrinogen is probably the most important factor involved in this hypercoagulable state, and high levels of this acute-phase protein are associated with cardiovascular disease (27) (Fig. 1). Plasma fibrinogen levels are known to increase in many infectious diseases, including C. pneumoniae and H. pylori infections (34), and research in our laboratories has revealed that fibrinogen levels and white cell counts are increased in patients with periodontal disease (24). Infections such as periodontal disease result in an increase in other acute-phase plasma proteins, which include C-reactive protein.

\section{General mechanisms by which infections may produce atherosclerosis}

\section{Monocyte-derived cytokines}

The proinflammatory cytokines produced by monocytes (IL-1, IL-6 and tumor necrosis factor $\alpha$ ) inhibit 
lipoprotein lipase and thus lipemia is a prominent feature of chronic infections (5) but also upregulates adhesion molecule expression on endothelial cells (13) and can stimulate mitogenesis and fibrinogen production. These cytokines only achieve high circulating levels in severe sepsis syndromes in which they are associated with pyrexia and fever. However, circulating plasma levels of IL-6 are also increased in smokers (25), correlating with the elevated fibrinogen levels and white cell counts in smokers. These findings may partly result from chronic oral (or lung) infections in smokers. As mentioned previously, monocyte infiltration into the subintima is a crucial pathogenic process in plaque development, and clearly the monocyte is crucial as both an infiltrating cell and a cell that can initiate the process by releasing cytokines, upregulating adhesion molecules and binding to them. The monocyte can also produce IL-8, a chemotactic factor that will aid in the recruitment of more leukocytes to this area.

\section{Periodontally specific mechanisms}

Periodontal disease is capable of predisposing to vascular disease given the abundance of gram-negative species involved, the readily detectable levels of proinflammatory cytokines in crevicular fluid, the dense immune cell infiltrates involved, the association of peripheral fibrinogen and white cell counts (24), and the extent and chronicity of this disease.

\section{Effect of lipopolysaccharide}

Infections produce changes in lipid metabolism which may favour atherosclerosis (43). As mentioned previously, proinflammatory cytokines such as tumor necrosis factor $\alpha$ and IL-1 inhibit lipoprotein lipase (5) and bacterial products such as lipopolysaccharide and muramyl dipeptide may have direct effects on endothelia such that atherosclerosis is promoted (36). Infections increase plasma fibrinogen levels, and these acute-phase proteins are significantly linked to atherosclerosis and also to infectious states, including the presence of periodontal disease.

Lipopolysaccharide is released as extracellular blebs from microorganisms within the periodontal pocket and may enter the diseased periodontium. It is unlikely that lipopolysaccharide will normally be free in the plasma due to efficient plasma protein binding. Bacteremia, however, or microorganism invasion may result in free lipopolysaccharide from periodontal organisms being present in the plasma. Bacteremia is considered possible following disturb- ance of the chronic lesion during activities such as scaling and during acute and chronic abscess conditions. Bacteremia will result in gram-negative organisms entering the circulation, where they may activate leukocytes, platelets or endothelium directly. These circulating microorganisms may also shed their lipopolysaccharide which will have a detrimental effect on lipid metabolism as outlined previously.

Lipopolysaccharide may bind to lipopolysaccharide-binding protein, a high-affinity plasma carrier protein (47). When lipopolysaccharide is bound to lipopolysaccharide-binding protein it is then able to bind to CD14 receptors, either soluble or on endothelium or on monocytes or macrophages, which would result in cellular activation. Cellular activation will produce upregulation of adhesion molecules following cellular cytokine and chemokine release. Subintimal leukocyte infiltration and proliferation of smooth muscle cells in the lesion may follow (Fig. 2). Fibrinogen and white cell count increases noted in periodontitis patients may be a secondary effect of the above mechanisms or a constitutive feature of those at risk of both cardiovascular disease and periodontitis (24) (such as smokers).

\section{Hyper-reactive mononuclear phagocytes}

Hyper-reactive mononuclear phagocytes may be constitutive in susceptible individuals or may be induced in patients who smoke or those with infections such as periodontal disease. These may be induced if leukocytes are passing through lesions in vessels traversing close to areas of high proinflammatory cytokine release, lipopolysaccharide presence, matrix metalloproteinase activity or prostaglandin or protease release. These leukocytes may, by virtue of their induced hyper-responsiveness, promote atherosclerosis at distant sites, particularly in areas of disturbed blood flow.

There is also the possibility that cells such as macrophages or Langerhans cells, which recirculate under normal circumstances, may themselves be activated and enter the circulation or may activate other peripheral circulation cells, and these indirectly activated cells may have an effect at distant sites. It has also been reported that peripheral polymorphonuclear leukocytes from periodontitis patients show more than two-fold higher chemiluminescence following Fc $\gamma$ receptor stimulation than healthy control polymorphonuclear leukocyte (14). This polymorphonuclear leukocyte hyperreactivity may be due to these cells moving in the circulation through periodontal lesions or may be a constitutive feature of pa- 
tients with periodontal disease. Whatever the mechanism, these cells may similarly be capable of aiding atheroma formation. Langerhans cells may directly bind lipopolysaccharide that has infiltrated through the epithelium or lipopolysaccharide on the membranes of invading bacteria may contact these or other long-lived circulating cells. Lipopolysaccharide coming into the tissues or circulation may bind to lipopolysaccharide-binding protein and this complex may bind to either soluble or cell-bound CD14 receptors and thus activate cells. If CD14 receptors on monocytes or endothelium are activated this may encourage atheroma formation, but even if soluble lipopolysaccharide-lipopolysaccharide-binding proteinCD14 aggregates occur, these may bind to the vasculature at some point and result in atherosclerotic lesion formation, probably at sites of disturbed blood flow, and this may go some way to explaining the predisposition of certain sites to atheromas. Interestingly, patients with a form of periodontal disease termed localized juvenile periodontitis have peripheral monocytes that, when reacting with lipopolysaccharide, give increased prostaglandin $\mathrm{E}_{2}$ production (43), but membrane-bound CD14 is not correlated with monocyte hyper-responsiveness to lipopolysaccharide. In vitro conditions, however, reverse the hypersensitivity of localized juvenile periodontitis monocytes to approximately control levels, and it is feasible that this hyper-responsiveness is induced by the persistent infection of the periodontium rather than being a constitutive feature of localized juvenile periodontitis monocytes. Thus, there appears to be evidence for periodontal disease inducing hyper-responsive leukocytes, and this may yet be shown to have a role in atheroma production and thus provide a mechanistic link between periodontitis and atheroma formation.

A further link between smoking, monocyte responsiveness and lipopolysaccharide stimulation is provided by Payne et al. (35), who demonstrated that lipopolysaccharide-mediated monocyte secretion of prostaglandin $E_{2}$ is increased by nicotine. Thus, smoking may have biological effects on both periodontal disease and atheromatous plaque formation through this mechanism.

Platelets and leukocytes may be activated during bacteremia and go on to excite other cells, enhancing the likelihood of atheromatous plaque formation. Indeed, it has been proposed that activated platelets may regulate chemokine release by monocytes in inflammatory lesions (49). Platelets are an integral part of the hemostatic processes, which are also crucial to atheroma formation, and further- more, these cells have been shown to be triggered by several dental microorganisms (16).

\section{Conclusions}

Periodontal and cardiovascular diseases share many risk factors and pathogenic processes. Infections and chronic inflammatory conditions such as periodontitis may influence the atherosclerotic process. The chronicity of periodontal disease provides a rich source of subgingival microbial and host response products and effects over a long time period. Two main processes may provide a causative link between these two diseases: the lipopolysaccharide and monocyte-related responses. Insufficient experimental evidence exists, however, to further support these hypotheses at present, and whether one or both of these processes transpire to be pivotal remains to be determined.

\section{References}

1. American Academy of Periodontology. Tobacco use and the periodontal patient. J Periodontol 1996: 67: 51-56.

2. Beck J, Offenbacher S, Williams R, Gibbs P, Garcia R. Periodontitis: a risk factor for coronary heart disease? Ann Periodontol 1998: 3: 127-141.

3. Benditt EP, Barrett T, McDougall JK. Viruses in the aetiology of atherosclerosis. Proc Natl Acad Sci U S A 1983: 80: 63866389.

4. Benditt EP, Benditt JM. Evidence for a monoclonal origin of human atherosclerotic plaques. Proc Natl Acad Sci U S A 1973: 70: 1753-1759.

5. Beutler BA, Cerami A. Recombinant interleukin-1 suppresses lipoprotein lipase activity in 3T3-L1 cells. J Immunol 1989: 135: 3969-3971.

6. Burke JM, Ross R. Synthesis of connective tissue macromolecules by smooth muscle. Int Rev Connect Tissue Res 1979: 8: 119-157.

7. Clarke NG, Shephard BC, Hirsch RS. The effect of intraarterial epinephrine and nicotine on gingival circulation. Oral Surg Oral Med Oral Pathol Oral Radiol Endod 1981: 52: $577-582$.

8. Collins R, Peto R, Baigent C, Sleight P. Drug therapy: aspirin, heparin and fibrinolytic therapy in suspected acute myocardial infarction. N Engl J Med 1997: 336: 847-860.

9. Cook PJ, Honeybourne D. Chlamydia pneumoniae. J Antimicrob Chemother 1994: 34: 859-873.

10. Cook PJ, Lip GYH. Infectious agents and atherosclerotic vascular disease. Quintessence J Med 1996: 89: 727-735.

11. Davies MJ, Thomas AC. Thrombosis in acute coronary artery lesions in sudden ischaemic death. N Engl J Med 1984: 310: 1137-1140.

12. Filler SG, Pfunder AS, Spellberg BJ, Spellberg JP, Edwards JE Jr. Candida albicans stimulates cytokine production and leukocyte adhesion molecule expression by endothelial cells. Infect Immun 1996: 64: 2609-2617. 
13. Gamble JR, Harlan JM, Klebanoff SJ. Stimulation of the adherence of neutrophils to umbilical vein endothelium by human recombinant tumor necrosis factor. Proc Natl Acad Sci U S A 1985: 82: 8667-8671.

14. Gustafsson A, Asman B. Increased release of free oxygen radicals from peripheral neutrophils in adult periodontitis after Fc delta-receptor stimulation. J Clin Periodontol 1996: 23: 38-44.

15. Gyorkey F, Melnick JL, Guinn GA, Gyorkey P, DeBakey ME. Herpesviridae in the endothelial and smooth muscle cells of the proximal aorta in arteriosclerotic patients. Exp Mol Pathol 1984: 40: 328-334.

16. Herzberg MA, Meyer MW. Dental plaque, platelets and cardiovascular disease. Ann Periodontol 1998: 3: 151-160.

17. Holt PG. Immune and inflammatory function in cigarette smokers. Thorax 1987: 42: 241-249.

18. Hull RD, Pineo GF, ed. Disorders of thrombosis. Philadelphia: WB Saunders, 1996.

19. Johnson JD, Houchens DP, Kluwe WM, Craig DK, Fisher GL. Effects of mainstream and environmental tobacco smoke on the immune system in animals and humans. A review. Crit Rev Toxicol 1990: 20: 369-395.

20. Kenney EB, Kraal JH, Saxe SR, Jones J. The effect of cigarette smoke on human oral polymorphonuclear leukocytes. J Periodontal Res 1977: 12: 227-234.

21. Klurfeld DM. Identification of foam cells in human atherosclerotic lesions as macrophages using monoclonal antibodies. Arch Pathol Lab Med 1985: 109, 445-449.

22. Kraal JH, Chancellor MB, Bridges RB, Bemis KG, Hawke JE. Variations in the gingival polymorphonuclear leukocyte migration rate in dogs induced by chemotactic autologus serum and migration inhibitor from tobacco smoke. J Periodontal Res 1977: 12: 242-249.

23. Kweider M, Lowe GDO, Murray GD, Kinane DF, McGowan DA. Dental disease, fibrinogen and white cell count; links with myocardial infarction? Scottish Med J 1993: 38: 73-74.

24. Lowe GDO, Rumley A, Yarnell JWG, Sweetnam PM. Fibrin D-dimer, von Willebrand factor, tissue plasminogen activator antigen, and plasminogen activator inhibitor activity are primary risk factors for ischaemic heart disease: the Caerphilly Study. Thromb Haemost 1995: 73: 950.

25. Lowe GDO, Yarnell JWG, Sweetnam PM, Rumley A, Thomas HF, Elwood PC. Fibrin D-dimer, tissue plasminogen activator, plasminogen activator inhibitor, and the risk of major ischaemic heart disease in the Caerphilly Study. Thromb Haemost (in press).

26. Lowe GDO. Agents lowering blood viscosity, including defibrinogenating agents. In: Verstraete M, Fuster V, Topol E, ed. Cardiovascular thrombosis-thrombocardiology. 2nd edn. Philadelphia: Lippincott Raven (in press).

27. Lowe GDO. Aetiopathogenesis of cardiovascular disease: haemostasis, thrombosis and vascular medicine. Ann Periodontol 1998: 3: 121-126.

28. McCullagh KE, Ballian G. Collagen characterisation and cell transformation in human atherosclerosis. Nature 1975: 258: 73-75.

29. McDonald K, Rector TS, Braunlin EA, Kubo SH, Olivari MT. Association of coronary artery disease in cardiac transplant recipients with cytomegalovirus infection. Am J Cardiol 1989: 64: 359-362.

30. Melnick JL, Dreesman GR, Mecollum CH, Petrie GL, Burek J, DeBakey ME. Cytomegalovirus antigen within human arterial smooth muscle cells. Lancet 1983: 2: 644-647.
31. Minick CR, Fabricant CG, Fabricant J, Litrenta MM. Atherosclerosis induced by infection with a herpes virus. Am J Pathol 1979: 96: 673-706.

32. Mitchinson MJ. Macrophages and atherogenesis. Lancet 1987: ii: $146-149$.

33. Patel P, Carrington D, Strachan DP. Fibrinogen: a link between chronic infection and coronary heart disease. Lancet 1994: 343: 1634-1635.

34. Patel P, Carrington D, Strachan DP. Fibrinogen: a link between chronic infection and coronary heart disease. Lancet 1994: 343: 1634-1635.

35. Payne JB, Johnson GK, Reinhardt RA, Dyer JK, Maze CA, Dunning DG. Nicotine effects on $\mathrm{PGE}_{2}$ and IL-1 beta release by LPS-treated human monocytes. J Periodontal Res 1996: 31: 99-104.

36. Pesonen E, Kaprio E, Rapola J, Soveri T, Oksanen H. Endothelial cell damage in piglet coronary artery after intravenous administration of $E$. coli endotoxin. Atherosclerosis 1981: 40: 65-73.

37. Psaty BM, Koepsell TD, Manolio TA, Longstreth WT, Wagner EH. Risk ratios and risk differences in estimating the effect of risk factors for cardiovascular disease in the elderly. J Clin Epidemiol 1990: 43: 961-970.

38. Raulin LA, McPherson JC, McQuade MJ, Hanson BS. The effect of nicotine on the attachment of human fibroblasts to glass and human root surfaces in vitro. J Periodontol 1988: 59: 318-325.

39. Rosenfeld ME, Tsukada T, Gown AM, Ross R. Fatty streak initiation in Watanabe heritable hyperlipemic and comparable hypercholesterolemic fat-fed rabbits. Arteriosclerosis 1987: 7: 9-23.

40. Ross R. The pathogenesis of atherosclerosis: a perspective for the 1990s. Nature 1993: 326: 801-809.

41. Saikku P, Leinonen M, Mattila K. Serologic evidence of an association of a novel Chlamydia, TWAR, with chronic coronary heart disease and acute myocardial infarction. Lancet 1988: ii: 983-986.

42. Sammalkorpi K, Valtonen V, Kerttula Y, Nikkila E, Taskinen MR. Changes in serum lipoprotein pattern induced by acute infection. Metabolism 1988: 37: 859-865.

43. Shapira L, Soskolne WA, Van Dyke TE. Prostaglandin $E_{2}$ secretion, cell maturation, and CD14 expression by monocyte-derived macrophages from localized juvenile periodontitis patients. J Periodontol 1996: 67: 224-228.

44. Smith EB. Fibrinogen and fibrin degradation products in relation to atherosclerosis. Clin Haematol 1986: 15: 355.

45. Sorlie PD, Adam E, Melnick SL. Cytomegalovirus herpesvirus and carotid atherosclerosis: the ARIC study. J Med Virol 1994: 42: 33-37.

46. Thompson WD, McGuigan CJ, Snyder C. Keen GA, Smith EB. Mitogenic activity in human atherosclerotic lesions. Atherosclerosis 1987: 66: 85-93.

47. Tobias PS, Gegner J, Tapping R, Orr S, Mathison J, Lee KJD, Kravchenko V, Han J, Ulevitch RJ. Lipopolysaccharide dependent cellular activation. J Periodontal Res 1997: 32; 99103.

48. Weyrich AS, Elstad MR, McEver RP, McIntyre TM, Moore KL, Morrissey JH, Prescott SM, Zimmerman GA. Activated platelets signal chemokine synthesis by human monocytes. J Clin Invest 1996: 97: 1525-1534.

49. Woolf $\mathrm{N}$. The distribution of fibrin within the aortic intima: an immunohistochemical study. Am J Pathol 1961: 39: 521532. 